RESEARCH REPORT

\title{
An investigation of risk factors for symptomatic osteoarthritis of the knee in women using a life course approach
}

\author{
J Dawson, E Juszczak, M Thorogood, S-A Marks, C Dodd, R Fitzpatrick
}

J Epidemiol Community Health 2003;57:823-830

See end of article for authors' affiliations ......................

Correspondence to: Dr J Dawson, OCHRAD, School of Health and Social Care, Oxford Brookes University, 44 London Road, Oxford OX3 7PD, UK; idawson@ brookes.ac.uk

Accepted for publication 22 November 2002

\begin{abstract}
Study objective: To explore risk factors for symptomatic knee osteoarthritis (OAK) in women, which included wearing high heeled shoes.

Design: Matched case-control study. Exposure information obtained by interview, included details about past footwear. Self reported weight and height data obtained representing when women left school, were aged 36-40 and were aged 51-55. Regular work activities (including periods when homemaker or carer), smoking, and hormone related data gathered using a life-grid.

Setting: Interviews in participants' homes. Cases identified from orthopaedic hospital surgical waiting list, controls identified by general practitioners (GPs)

Participants: Women aged 50 to 70 years. Cases awaiting knee replacement surgery. Controls, who had no past knee surgery and no knee pain in the past three years, were matched for age and GP. Altogether 111 eligible women were interviewed (29 cases, 82 controls).

Main results: Univariate analyses identified several significant associations with OAK including past knee injury, arthritis of the feet, heavy smoking, being overweight (BMI 25 or above) and various occupational activities. Virtually all measures of high heeled shoes use were associated with reduced risk of OAK, although none of these findings were statistically significant. In multivariate analysis only BMI 25 or above at age 36-40 remained significantly associated with OAK (OR $36.4,95 \% \mathrm{Cl} 3.07$ to $432, p=0.004$ ), although weak evidence suggested certain occupational activities might increase risk. Weight gain in early adult life was particularly pronounced among cases.

Conclusions: Being overweight before the age of 40 considerably increased the risk of subsequent symptomatic OAK in women. Wearing high heeled shoes did not.
\end{abstract}

A round $2.4 \%$ of people over 55 years are disabled by knee pain due to symptomatic osteoarthritis of the knee (OAK). ${ }^{1}$ The prevalence of OAK increases with age, with a greater increase in women. By the age of 65 years, around twice as many women as men are affected. This gender difference suggests that women either carry a higher risk of OAK in itself, or that they experience greater exposure to risk factors. ${ }^{23}$

A number of risk factors for OAK have been identified in studies using a variety of case definitions. These include: particular genotypes-implicated in a minority of cases, ${ }^{4}$ obesity, ${ }^{5}$ extreme levels of exercise, ${ }^{7-10}$ knee joint injury, ${ }^{11-13}$ and various occupational factors. ${ }^{14}{ }^{15}$ Other risk factors that have been suggested include smoking (as a "protective" influence), ${ }^{6}$ oestrogen-that is, oral contraception or replacement therapy (HRT) (here, the evidence is particularly inconsistent), ${ }^{16}{ }^{17}$ and factors that relate to social class. ${ }^{18-20}$

It has been suggested that the greater prevalence of OAK among women might be related to the wearing of high heeled shoes, ${ }^{21} 22$ although the evidence is limited. Wearing high heels has become increasingly common since the first world war, and any risk associated with such footwear should be quantified. We have been unable to identify any epidemiological studies that evaluate this potential risk factor, and therefore our aim was to explore this issue with the pilot study described here.

The proposed lead time between the wearing of high heels and the onset of OAK is long (several decades), so a casecontrol study was the only practical study design to use for this investigation. However, this presented the problem of measuring past exposure, and we decided to use a life course approach.
The life course approach seeks to examine the relation between individuals' health and their changing social conditions over time. It aims to assist recall by using cognitive tools based on the respondent's own life course or "life events calendar". We adopted a form of the approach where a "life grid" is used as part of an interview technique. ${ }^{23}$ This method has been shown to minimise recall bias after as long as 50 years. ${ }^{23}$

We report here the risk factor relations observed in the feasibility study that was undertaken to test our methodology.

\section{METHODS}

The study obtained approval from the Central Oxford Research Ethics Committee (reference C99.157).

\section{Study subjects}

Women aged 50 to 70 years resident within 20 miles of a specialist orthopaedic hospital were studied.

\section{Cases}

Cases were defined as women who reported at least moderate knee pain on most days in the past month and had been placed on a waiting list within the preceding 12 months for a primary unicompartmental or total knee replacement (TKR), with primary idiopathic OA as the indication for surgery. Cases were identified from a consultant's (CD) surgical waiting list. Details of the patient's indication for surgery and

Abbreviations: OAK, osteoarthritis of the knee; HRT, hormone replacement therapy; $\mathrm{BMI}$, body mass index 
inclusion/exclusion criteria were verified using hospital medical notes. Potential cases were sent a letter of invitation signed by their hospital consultant, together with an explanation of the study. One week later the research nurse telephoned the patient to obtain their response and arrange an interview.

\section{Controls}

Controls were sought from the general practice with which each case was registered and were matched for age (within two years). The local ethics committee required that potential controls were selected by the general practitioner rather than the research nurse. Women who reported regular moderate knee pain or who had discussed a knee problem with a doctor within the past three years were excluded by the GP (with reference to patients' notes) or-if the GP was unaware of this problem-by standard screening questions asked at a later stage. We approached all GPs in Oxfordshire at the start of the study, explaining the purpose of the study and the role that they would be asked to play. Fifty two GPs (52 of 389, $13 \%$ ) indicated at this stage that they were not willing to participate. When a case had been interviewed, a letter was sent to the GP requesting the identification of six age matched controls and stating the inclusion/exclusion criteria. The research nurse provided customised letters of invitation for the general practitioner to sign and post.

Reminders were sent to all non-responders after two weeks, even where one or more women within a case-control set had already agreed to participate, and all women who agreed to participate were then selected as controls. The research nurse then telephoned selected controls to arrange an interview, and asked some standardised questions to check the eligibility of the woman.

\section{Exclusion criteria}

Cases and controls were excluded if any of the following factors were present, either reported by the patient or recorded in the medical notes:

- Previous other surgery for OA (for example, osteotomy, TKR), on either knee.

- A diagnosis of rheumatoid arthritis or other generalised inflammatory condition of the joints.

- Genetic or metabolic predisposition to secondary OA or other joint disease, for example, a diagnosis of psoriasis, haemophilia, or Paget's disease.

\section{Sample size}

We took a pragmatic approach to the sample size, rather than carrying out a formal power calculation. No population data were available relating to women's history of high heel use, and this study was intended primarily to be a feasibility test of our methods. We aimed to obtain as many matched case/ control sets as possible in the 10 month recruitment phase, and accepted that the study would have low power, but would provide valuable information that would facilitate the powering of a future main study.

\section{The interview format and schedule}

Interviews took place in the respondents' own homes. Respondents were asked in advance if they could have available photographs of themselves, preferably full length, taken over their adult life. Other picture cues were provided by the research nurse.

Interviews began by identifying dates of significant personal events, and lifetime information on residence and occupation. This information was entered on a life grid and details of part time and full time jobs and periods of unemployment or home-making were recorded. As the life grid evolved, items of information were simultaneously recorded into standard tables. This method permitted the calculation of "total number of years spent-for instanceliving in houses without central heating, or doing work that involved regular lifting, or wearing shoes with 2 inch heels". Part time jobs were counted as half time in all cases. Once completed, the life grid was used as a cross referencing aid to recall.

The interview next focused on potential risk factors including: occupational activities (collected for each period of work-or period spent as home-maker, mother or carerthat lasted for one year or more); past shoe wearing behaviour; participation in competitive sports; past and present problems affecting any joints; height, body weight at three different stages of life, cigarette smoking, child bearing, and use of contraceptive pills or hormone replacement therapy.

\section{Measurement of potential risk factors Occupational risk factors}

In relation to each job already recorded (which also included periods when the respondent was a "home-maker, mother or carer"), respondents were asked: "Did this job/period in your life involve any of the following activities on a regular basis (at least two days per week)?" Activities were then listed and included: lifting, kneeling, bending, squatting, or walking for long periods. Respondents were also asked if the job/period in their life had specifically involved regularly cleaning floors on their knees. This information, together with information on the length of time spent in each "period of work", was used to compute the total numbers of years spent doing work that involved each of the named activities.

\section{Body weight and body mass index}

Respondents reported their height and typical weight around the time they left school, were aged 36-40, and were aged 5155 years. Body mass index (body weight in kilograms/height in metres $^{2}$ ) was computed for each juncture.

\section{Smoking}

Respondents were asked about all periods when they had smoked and the total number of cigarettes smoked in the woman's lifetime was calculated. This was then categorised as never smoked, moderate or heavy smoker. A heavy cigarette smoker was defined as a woman who had smoked more than 70000 cigarettes. This is equivalent to just over five cigarettes per day for 40 years or 20 cigarettes per day for 10 years.

\section{Past shoe wearing behaviour}

The following measures of shoe wearing behaviour were collected:

(a) Respondents were asked to say how old they were when they first wore a pair of shoes with heels one, two or three inches high-if ever.

(b) A file of pictures of 38 different styles and heights of shoes, with both front and side view was used. In each case, the facing page showed life size pictures of heels with a range of different heights. These were labelled " $\mathrm{A}$ " to "I". Respondents were asked to say if they had ever worn regularly any shoes similar to each of the pictures shown for work, for going out socially or for dancing, and, if so, to identify from the pictures the most likely heel height in each case. The highest heel recorded for each five year period for each type of use was used in the analysis, and contributed to six derived variables giving the total number of years between age 10 and 40 years when the subject had regularly 
worn heels higher than two inches and heels higher than three inches, for work, for going out socially, or for dancing.

\section{Statistical analysis}

We used conditional logistic regression to compare cases and controls in relation to the frequency of risk factors for all univariate and multivariate analyses using STATA software (release 7.0 ).

\section{RESULTS}

\section{Participation}

Fifty nine cases were identified, and 31 (53\%) were interviewed. A median of three controls per case were interviewed (range 1 to 6 ). One hundred and eleven women (29 cases, 82 controls) were both interviewed and eligible. See figure 1.

\section{Sample characteristics and risk factors}

Table 1 presents descriptive data together with odds ratios from univariate analyses comparing cases and controls. There were no significant differences in any of the measures of socioeconomic status between cases and controls. However, the most highly statistically significant result was body mass index (BMI) when aged 36-40. A significantly higher proportion of cases than controls reported a BMI of 25 or above (BMI 25 has been used as a threshold for being classified as overweight, but more recently the nomenclature

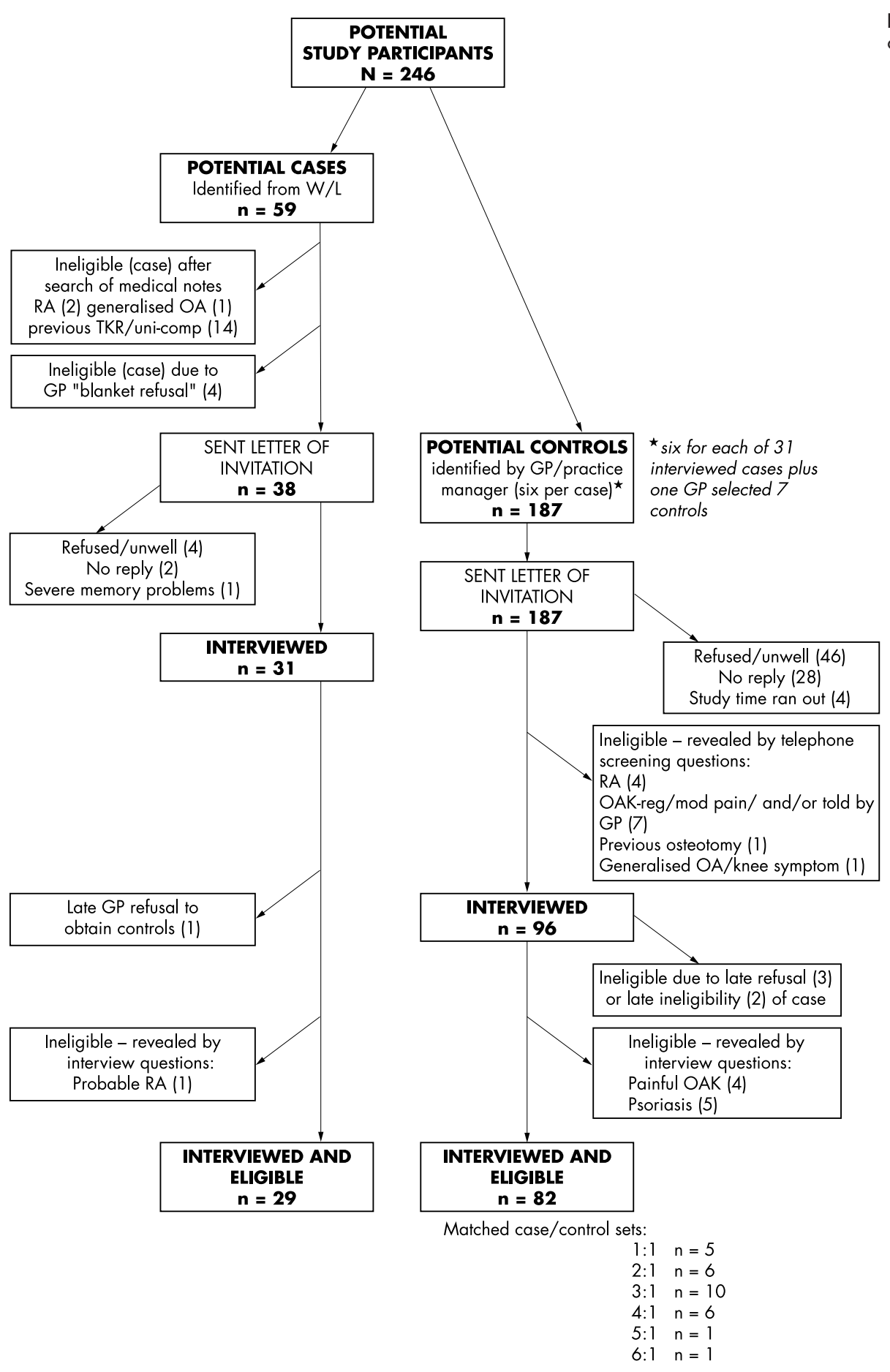

Figure 1 Selection of cases and controls and reasons for their exclusion. 


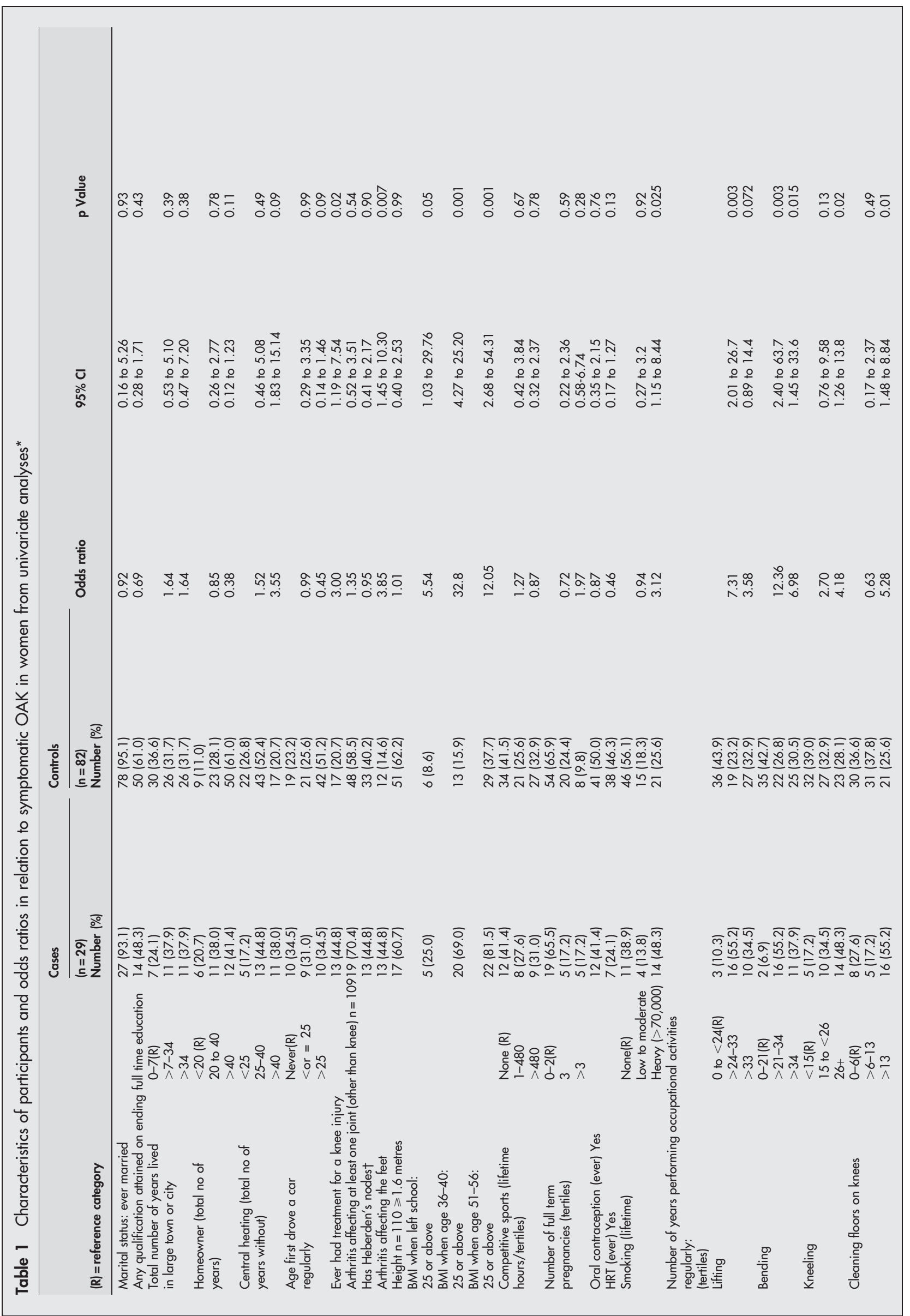




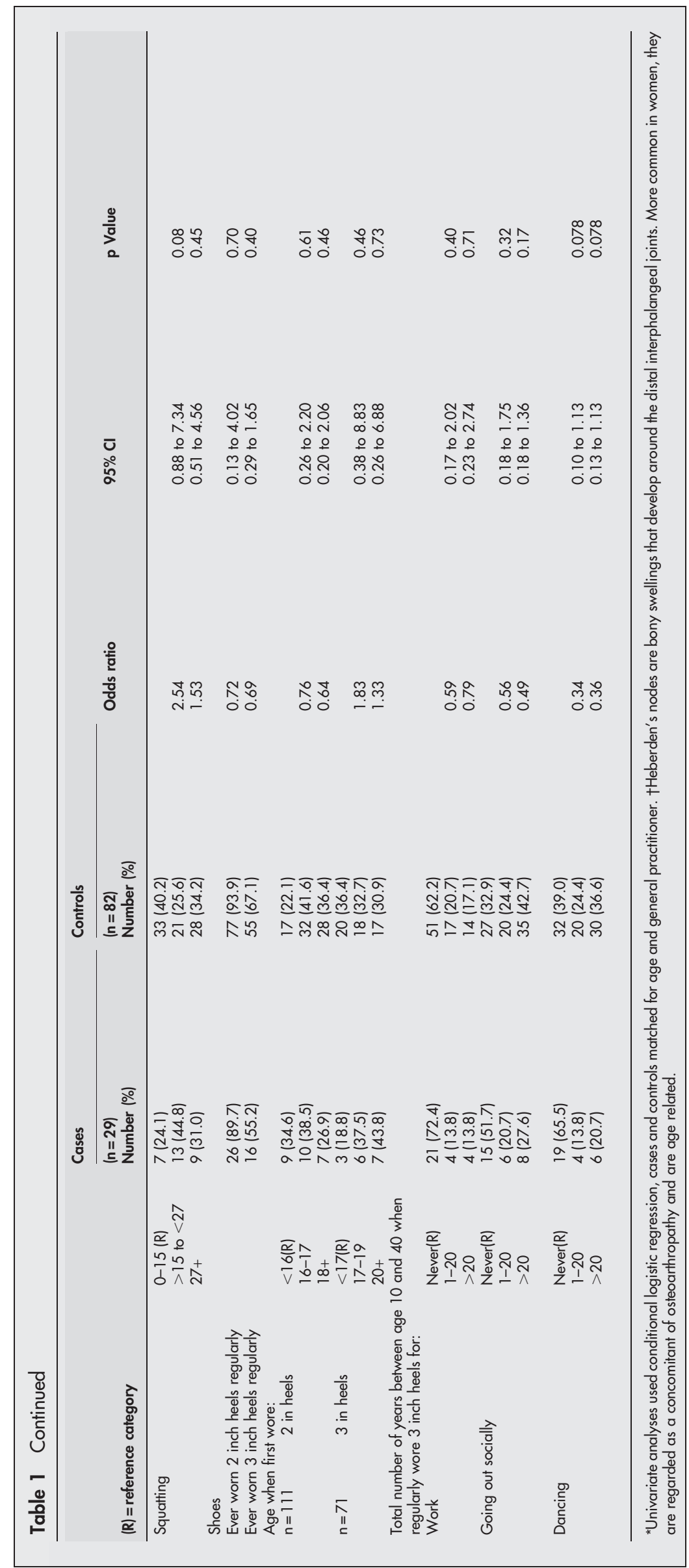




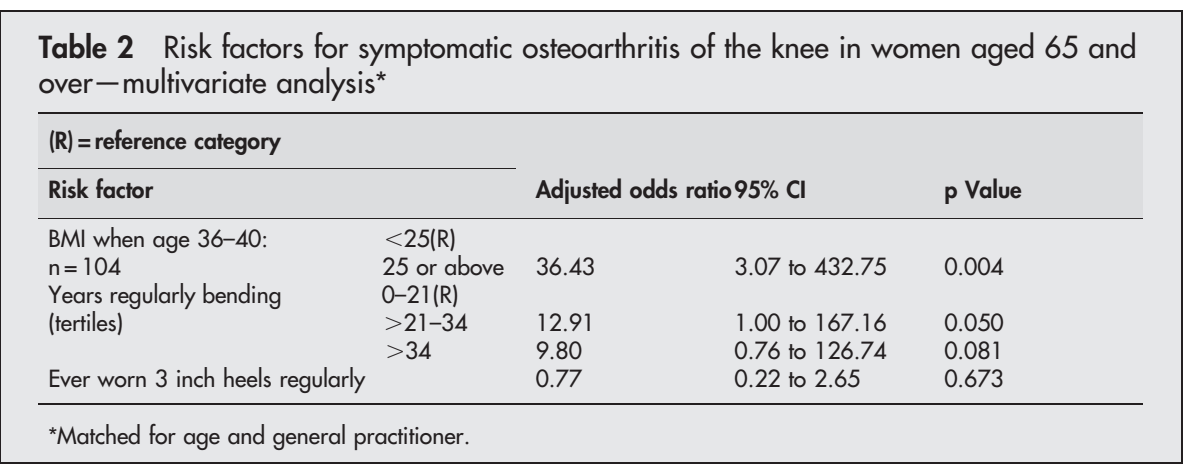

has changed to pre-obese ${ }^{24}$ ) when they were $36-40$ years of age, (cases: 20 of 29, 69\%; controls: 13 of $82,16 \%$; OR 32.8, $95 \%$ CI 4.3 to $252, p=0.001)$. Cases reported greater numbers of other problems with their knees and feet, but this may be an artefact of the exclusion of controls who had consulted with their doctor regarding knee problems in the past three years, and also the fact that cases will have had greater access to diagnostic facilities. Heavy smoking was associated with a threefold increase in risk of OAK.

Most respondents had spent many years doing physically demanding work. Several activities showed a significant relation with OAK, in particular lifting and bending. However, there was no clear exposure "dose-response" with increasing years. All respondents reported wearing shoes with heels at least one inch high at some time in their lives, while 8 of $111(7 \%)$ said that they had never worn heels as high as two inches and 40 of 111 (36\%) reported never wearing three inch heels. None of the measures of high heel wearing were significantly associated with OAK and in most cases the odds ratios indicated a protective effect rather than the hypothesised increase in risk. There were no significant differences in parity, competitive sports activity, use of oral contraceptives, or use of hormone replacement therapy.

\section{Multivariate analysis}

To study further the interrelation between OAK and either established or suspected risk factors (including those approaching conventional levels of statistical significance in the univariate analysis - that is, $\mathrm{p}<0.05$ ), we performed a multivariate analysis using conditional logistic regression, using matched case-control sets. A number of models were tested with variables added systematically to a baseline model containing a binary variable for BMI aged 36-40 (25 or above, versus $<25)$. Factors that exhibited a weak association with OAK $(\mathrm{p}<0.1)$ independent of, and in addition to the effect of BMI aged 36-40, remained in the model. One notable exception to this rule was the factor "ever wore heels over three inches". This was entered as it was our hypothesis that high heel wearing would be a harmful activity and therefore we sought to quantify the risk of OAK. We chose this particular variable because women generally underestimated the height of heels by around half an inch (unpublished data) and so we felt that this variable would best represent high heels. We examined the known/likely risk factors for OAK, plus those other variables significantly associated with OAK in univariate analysis and excluded any measure of self reported foot arthritis or knee injury in the final model for reasons already mentioned.

Factors denoting occupational activity were highly correlated and we therefore chose to enter just one of these variables, "years (tertiles) regularly bending" in the model. Bending was highly correlated with most of the other occupational factors and was only weakly associated with OAK independently of BMI aged 36-40, and its inclusion in the multivariate model did not destabilise it. The inclusion of a variable relating to smoking made the model highly unstable; it was not significantly associated with OAK independently of BMI aged 36-40 and as we had a comparatively small sample size and thus could not expect to have discriminatory power over many variables it was excluded from the model. We chose to use BMI at age 36 to 40 years because we felt the later BMI (at age 51 to 55 ) could be confounded by the relative immobility of women with OAK, in that knee pain discourages activity and thereby encourages weight gain.

The final model therefore included BMI greater than $25 \mathrm{~kg} / \mathrm{m}^{2}$ at age 36 to 40 years, years bending within occupation, and wearing three inch heels (ever/never). Results of the final multivariate analysis are shown in table 2. Only a self reported BMI of 25 or above at around the age of 36-40

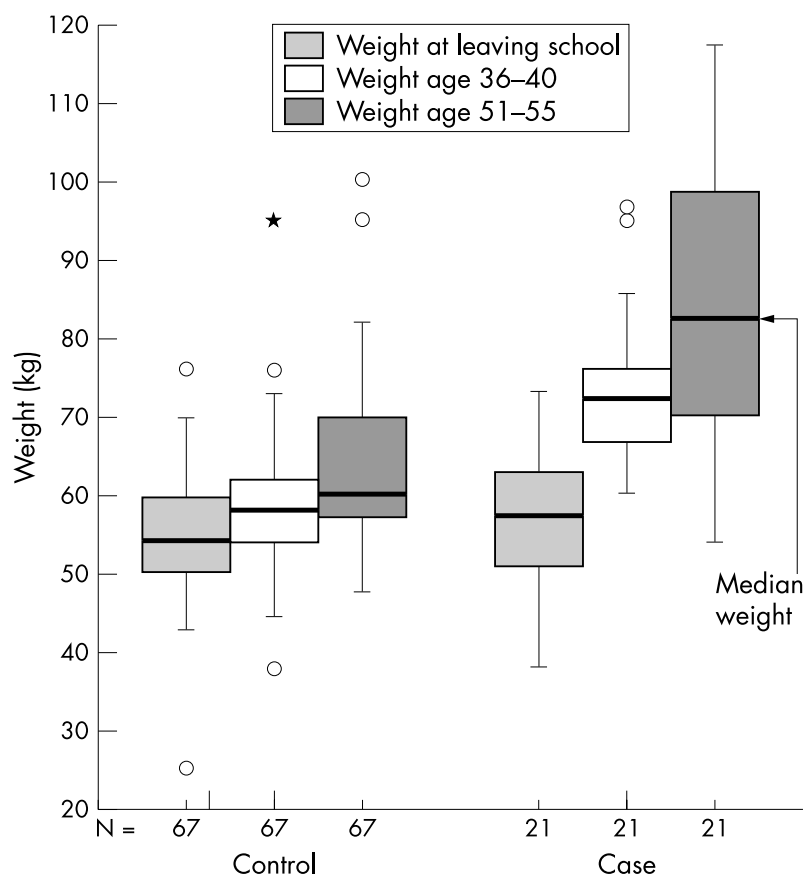

Figure 2 Boxplot comparing cases' and controls' reported body weight at three stages of life: when they left school, during their late 30s, and during their early $50 \mathrm{~s}$. Median change $(\mathrm{kg})$ between leaving school and age period 36 to 40 : cases: 12.7 , ranging from -1.8 to 50.5 compared with controls: median change $(\mathrm{kg}) 3.2$, ranging from -15.9 to 35.0 ; $\mathrm{p}=0.004 \uparrow$. Median change $(\mathrm{kg})$ between age period 36 to 40 years and age period 51 to 55 years (median change $(\mathrm{kg})$ : cases: 6.4 ranging from -12.7 to 44.1 compared with controls: median change $(\mathrm{kg})$ : 5.0 , ranging from -3.2 to $22.3 ; p=0.3+$. + Test of statistical significance Wilcoxon signed rank test, using median value of controls within each matched case/control set. 
Key points

- It is very unlikely that prolonged wearing of high heeled shoes represents a risk factor for symptomatic osteoarthritis of the knee (OAK) in women.

- Attaining a body mass index of 25 or above before the age of 40 seems to increase the risk of symptomatic OAK in women markedly.

- Future research in relation to risk and prevention of OAK might usefully focus on the age at which people first undergo excessive weight gain and whether or not this gain is sustained.

years remained highly significantly associated with having OAK (OR 36.4, 95\% CI 3.07 to $432, p=0.004$ ), although there was some weak evidence that regular bending in the course of occupational activities might be associated with an increased risk of OAK. The wide confidence interval represents the inherent uncertainty of our estimates, both because of low power and therefore precision, and measurement error, as weight was self reported.

Figure 2 presents a boxplot comparing cases and controls' reported body weight at three stages of life: around the time that they left school, during their late 30s and during their early 50s. The change in weight that occurred between leaving school and the age period 36 to 40 years was significantly greater for the cases than for the controls $(p=0.004)$, and was also much greater than that which occurred during the period between age 36 to 40 years and age 51 to 55 years $(\mathrm{p}=0.3)$.

\section{DISCUSSION}

We have described a feasibility study using established casecontrol methodology, to investigate risk factors for symptomatic OAK in women. A key aim was to test methods for obtaining detailed exposure information on risk factors, (particularly the past use of high heeled shoes), which has not systematically been obtained before. Population based data of this type were not available before this study so that a formal sample size calculation was not appropriate and a large scale project could not be justified in this instance. We therefore adopted a pragmatic approach, which resulted in a small sample and this will have limited the power of the study to detect or confirm risk factors in our analysis. In addition, a small sample size coupled with a large number of risk factors studied increases the risk of obtaining false positive results and the findings should therefore be treated with due caution. We nevertheless obtained results that we believe to be of value, particularly in relation to the design of future studies of this type.

Data were gathered using a life course approach and interviews, with visual cues to aid recall. Commonly used socioeconomic indicators (for example, income, occupational classification) can change throughout a person's life and the measurement and meaning of such factors can be problematic_particularly in relation to women. ${ }^{25}$ The use of life course approaches to data collection offers ways of addressing this problem by, for instance, counting the number of years of exposure to particular variables, such as central heating. Results suggested that control subjects were generally of higher socioeconomic group than cases (fewer lifetime number of years in housing without central heating and more years of home ownership in controls than cases). However, these differences were not significant.

In common with one recent study, ${ }^{13}$ we could find no evidence of an association between sports related exercise and symptomatic OAK. This relation is still contentious, with some studies finding a relation. Receiving treatment for a past knee injury has been shown to increase the odds of subsequent OAK. ${ }^{13}{ }^{26}$ Our own study found this in univariate analysis (OR $3.0 \mathrm{p}=0.02$ ), however, controls were excluded if they had moderate knee pain or had consulted about a knee problem within the past three years, and this difference should be interpreted with extreme caution.

Occupational factors, which focused on the type of regular work activities performed, and which included periods when the women were home-makers or looking after their children, seemed to be related to risk of OAK, but this relation was no longer significant after controlling for BMI at age 36 to 40 years.

In contrast with other studies, ${ }^{6}$ we did not find any evidence in support of cigarette smoking as a protective factor for OAK in women. Indeed, univariate analysis suggested that heavy smokers were at a significantly increased risk compared with non-smokers. We also found no evidence in support of a protective effect of using HRT.

We examined various measures of past footwear exposure-particularly regarding the height of heels. Most of the women had been exposed to high heeled shoes over many years. Nevertheless, a consistent finding in univariate analyses was a reduced risk of OAK in association with regular high heel usage, although only one item (years of regular dancing in three inch high heels) was of statistical significance. None of the items were statistically significant after adjustment.

This surprising finding could be explained by measurement error in estimating exposure to wearing high heels, or it could be attributable to confounding by factors, particularly socioeconomic, that are related to high heel wearing. Nevertheless, our comparisons were underpowered and it therefore remains possible, although unlikely, that a larger study could find a relation between wearing high heels and increased risk of OAK - that is, we would not expect a large study to overturn the direction of this study's findings. A larger scale study would have to control for the very strong effect of weight/BMI and it is not obvious by what method this could be achieved, particularly if reliance was placed on self reports. Statistical adjustment is one option, but that did not particularly help us because the overlap in BMI was minimal between the two groups. No large scale study of this subject is planned by ourselves in the near future therefore.

In common with other research, we found that being overweight increased the risk of symptomatic OAK in women markedly. Nevertheless, while more women than men, in England, are classified as obese (21\% versus $17 \%$ respectively in 1998) a higher proportion of men than women are classified as pre-obese (BMI > 25). ${ }^{27}$ The considerably higher rates of OAK that are found in older women relative to men still, therefore, remains unexplained. Our own data suggest that future research in relation to risk and prevention of symptomatic OAK might usefully focus on the age at which people first undergo excessive weight gain and whether or not this gain is sustained.

\section{ACKNOWLEDGEMENTS}

The study was funded by the NHS Executive (south-east). The study received generous assistance from David Blane, Reader in Medical Sociology, Imperial College of Science, Technology and Medicine; Richard Edwards, Lecturer in Epidemiology and Public Health, Department of Epidemiology and Public Health, University of Newcastle upon Tyne; Sue Constable, Keeper of the boot and shoe collection, Northampton Museums and Art Gallery. We should also like to thank GPs and practice managers for their assistance and in particular, we would wish to thank all the women who took part and who welcomed our research nurse/interviewer into their homes. 


\section{Authors' affiliations}

J Dawson, Oxford Centre for Health Care Research and Development (OCHRAD) School of Health and Social Care, Oxford Brookes University, Oxford, UK

E Juszczak, Centre for Statistics in Medicine, Institute of Health Sciences, Oxford, UK

M Thorogood, Warwick Medical School, University of Warwick, Coventry, UK

S-A Marks, Division of Public Health and Primary Care, Department of Public Health, Institute of Health Sciences, Oxford, UK

C Dodd, Lower Limb Unit, Nuffield Orthopaedic Centre, Oxford, UK R Fitzpatrick, Division of Public Health and Primary Care, University of Oxford, UK

\section{REFERENCES}

1 Tennant A, Fear J, Pickering A, et al. Prevalence of knee problems in the population aged 55 years and over: identifying the need for knee arthroplasty. BMJ 1995;310:1291-3

2 Croft P. Review of UK data on the rheumatic diseases - 3. Osteoarthritis. BrJ Rheumatol 1990;29:391-5.

3 McAlindon TE, Cooper C, Kirwan JR, et al. Knee pain and disability in the community. Br J Rheumatol 1992;31:189-92.

4 Keen RW, Hart DJ, Lanchbury JS, et al. Association of early osteoarthritis of the knee with a Taq I polymorphism of the vitamin D receptor gene. Arthritis Rheum 1997:40:1444-9.

5 Hart DJ, Spector TD. The relationship of obesity, fat distribution and osteoarthritis in women in the general population: the Chingford Study. J Rheumatol 1993:20:331-5.

6 Felson DT. The epidemiology of knee osteoarthritis: results from the Framingham Osteoarthritis Study. Semin Arthritis Rheum 1990;20:42-50.

7 Panush RS, Hanson CS, Caldwell JR, et al. Is running associated with osteoarthritis? An eight year follow-up study. J Clin Rheumatol 1995; 1:35-9.

8 Spector TD, Harris PA, Hart DJ, et al. Risk of osteoarthritis associated with long-term weight-bearing sports: a radiologic survey of the hips and knees in female ex-athletes and population controls. Arthritis Rheum 1996;39:988-95.

9 Lequesne MG, Dang N, Lane NE. Sport practice and osteoarthritis of the limbs. Osteoarthritis Cartilage 1997:5:75-86.

10 McAlindon TE, Wilson PW, Aliabadi P, et al. Level of physical activity and the risk of radiographic and symptomatic knee osteoarthritis in the elderly: the Framingham study. Am J Med 1999;106:151-7.
11 Roos H, Adalberth T, Dahlberg L, et al. Osteoarthritis of the knee after injury to the anterior cruciate ligament or meniscus: the influence of time and age. Osteoarthritis Cartilage 1995;3:261-7.

12 Woo SL, Lewis JL, Suh J, et al. Acute injury to ligament and meniscus as inducers of osteoarthritis. In: Kuettner KE, Goldberg VM, eds. Osteoarthritic disorders. Rosemont: American Academy of Orthopaedic Surgeons, 1995: 185-96.

13 Sutton AJ, Muir KR, Mockett S, et al. A case-control study to investigate the relation between low and moderate levels of physical activity and osteoarthritis of the knee using data collected as part of the Allied Dunbar National Fitness Survey. Ann Rheum Dis 2001;60:756-64.

14 Coggon D, Croft P, Kellingray S, et al. Occupational physical activities and osteoarthritis of the knee. Arthritis Rheum 2000;43:1443-9.

15 Felson DT, Hannan MT, Naimark A, et al. Occupational physical demands, knee bending, and knee osteoarthritis: results from the Framingham Study. J Rheumatol 1991; 18:1587-92.

16 Felson DT, Nevitt MC. The effects of oestrogen on osteoarthritis. Current Opin Rheumatol 1998;10:269-72.

17 Zhang Y, McAlindon TE, Hannan MT, et al. Estrogen replacement therapy and worsening of radiographic knee osteoarthritis: the Framingham Study. Arthritis Rheum 1998;41:1867-73.

18 Gibson T, Hameed K, Kadir M, et al. Knee pain amongst the poor and affluent in Pakistan. Br J Rheumatol 1996;35:146-9.

19 Chaturvedi N, Ben Shlomo Y. From the surgery to the surgeon: does deprivation influence consultation and operation rates? Br J Gen Pract 1995; 45:127-31.

20 Leigh JP, Fries JF. Correlations between education and arthritis in the 19711975 NHANES I. Soc Sci Med 1994;38:575-83.

21 Kerrigan DC, Todd MK, Riley PO. Knee osteoarthritis and high-heeled shoes. Lancet 1998;351:1399-401.

22 Adrian MJ, Karpovich PV. Foot instability during walking in shoes with high heels. The Research Quarterly 1966;37:168-75.

23 Berney LR, Blane DB. Collecting retrospective data: accuracy of recall after 50 years judged against historical records. Soc Sci Med 1997;45: 1519-25.

24 Flegal KM, Carroll MD, Kuezmarski RJ, et al. Overweight and obesity in the United States: prevalence and trends, 1960-1994. Int J Obes Relat Metab Disord 1998;22:39-47.

25 Bartley M. Measuring women's social position: the importance of theory. J Epidemiol Community Health 1999;53:601-2.

26 Jensen $\mathrm{CH}$, Rofail S. Knee injury and obesity in patients undergoing total knee replacement: a retrospective study in 115 patients. J Orthop Sci 1999:4:5-7.

27 National Audit Office. Tackling obesity in England. London: The Stationery Office, 2001 